MaineHealth: Development, Implementation, and Success in Telehealth for Home Care

Robert Abel

Editor's note: On March $16^{\text {th }}$ and $17^{\text {th }}, 2017$, Telehealth and Medicine Today convened a national conference of opinion leaders to discuss and debate "Technologies and Tactics Transforming Long-term Care." What follows is the lecture by Robert Abel who is the Chief Nursing Officer and Director of Palliative Care for MaineHealth Care at Home, a member of the MaineHealth system.

\title{
Introduction [00:00]
}

Coming to the table and saying we are from Maine, people may not see that as applicable. So, I thought it would be good to kind of give you context about the scope of MaineHealth and what we do.

We've been doing telehealth since 2001, including several federal demonstration projects, looking mostly at heart failure. We had some success, but most of the time, and I think we've heard a couple speakers say this, a lot of this is just throwing technology out, magically collecting numbers, recording them someplace, and imagining that somehow patients are going to get better and not getting any worse.

\section{What would you say were your challenges? [00:39]}

There were lots of successes, but I think it's important to know there were a bunch of challenges; and many of these challenges still exist. Many of them you already heard about today: things like partnerships failing, people not being able to agree on the objective of the program, third party reimbursement still pending, and that continues to today. What we really wanted to do in 2017, and we did a strategic plan two years ago, is really impact care and really look at what we were doing.

We decided we weren't going to worry about reimbursement (Slide 1). We have HRS (Health Recovery Solutions) for our telehealth, and I'm going to say a couple of 
commercial names. I have no commercial interest, but I think it's important to know that there's a certain set of technologies that we're using. The other thing is we're using Epic for our medical records system.

\section{4 - 2017 Telehealth Aims:}

1) To expand access to care and improve quality measures for elders with at-risk chronic diseases by deploying enhanced telehealth technology

2) To expand access to care and improve quality measures for medically fragile children copying with the challenges of chronic disease or serious illness by introducing new pediatric telehealth modules and peripheral devices

3) To standardize protocols and integrate delivery of services on a system level

4) To improve coordination of care and communication with healthcare providers by securing an HL7 interface between EPIC and telehealth provider

MaineHealth

CARE AT HOME

Slide 1: MaineHealth telehealth aims.

\section{How would you describe your system? [01:25]}

We built interfaces specifically from our HRS (Health Recovery Solutions) tablets in the patient's home to the medical record; and we've produced alerts in the medical record to the providers. Literally, within 60 seconds of the patient's readings the provider can get an alert that the patient is out of parameter. That's not the program but I just want you to know that.

They are $4 \mathrm{G}$ tablets that the patient uses (Slide 2). There's a variety of data that we can input into the tablet, and we customize it to patients. 
These are some of the peripherals we typically use: blood pressure cups, wireless scales - that's all Bluetooth (Slide 3). We do a fair amount of pediatrics with this, which is a separate program. I'm not really going to talk about it, but just to let you know that we do that and we do pulse oximetry.

\section{Technology Highlights}
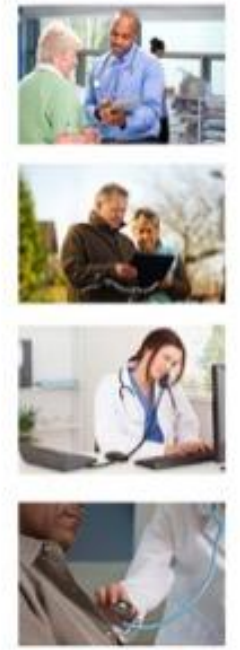

HRS
- $4 \mathrm{G}$ tablet with pre-loaded HRS software is provided at discharge. Automatic Setup can be achieved through EHR Integration

- Patients use tablet to comply with treatment plan \& remain engaged

- Data input to track activity, diet, weight \& medication available for clinician \& caregiver review

- Caregivers \& clinicians receive alerts to intervene if patient is at increased risk for readmission

Slide 2. Technology in telehealth. 


\section{The Peripherals}

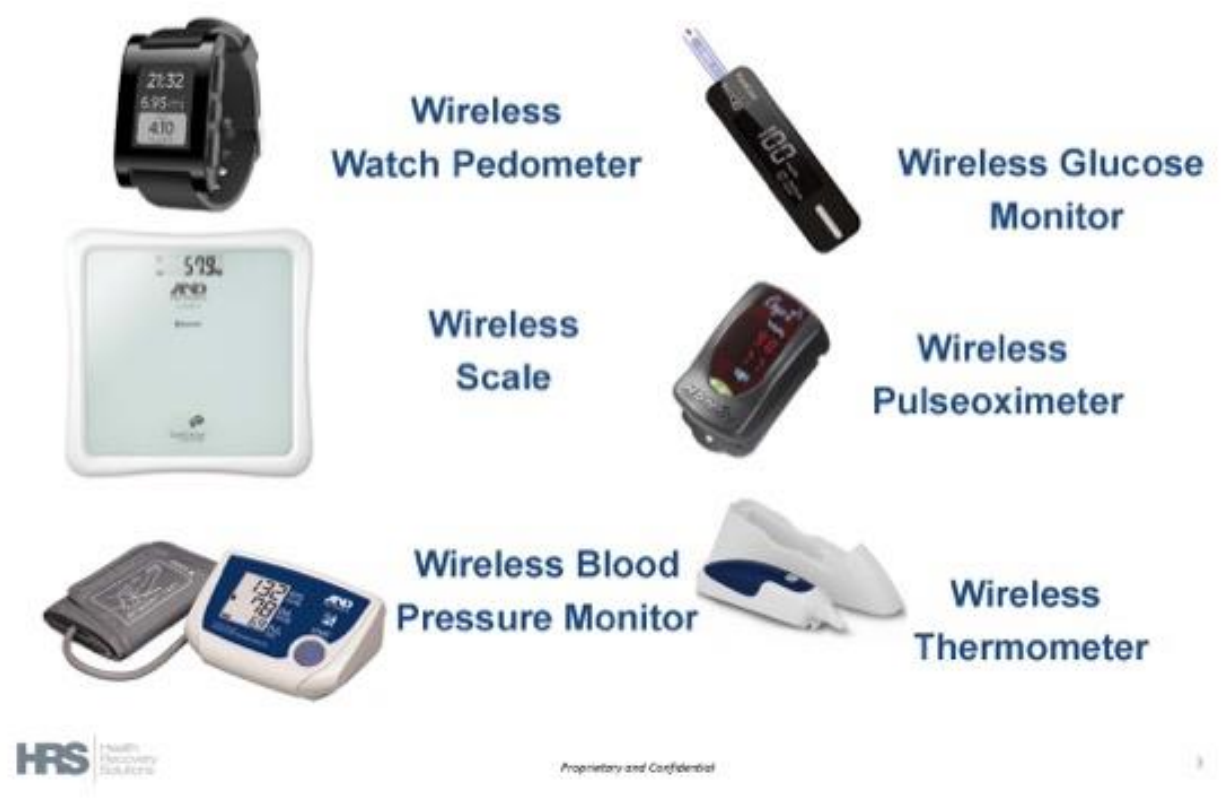

MaineHealth

Slide 3. Peripherals in telehealth.

\section{How large is MaineHealth? [02:14]}

What you should know is our ACO (Accountable Care Organization) is around the tenth or eleventh largest ACO in the country. MaineHealth takes care of about 1.2 million people a year across the system. The agency has an average daily census of about fifteen hundred patients, and that includes home health, private duty care and hospice. We're a very large agency, one of the five largest agencies in New England and typically benchmark against other agencies in New England.

Patient Connect is the system, and it actually talks to the patient. As it's telling the patient to weigh themselves or take their blood pressure, and those sorts of things. 
We also have educational videos, and we've been able to personalize that, so we actually have MaineHealth-created material that we upload, and part of that is longitudinal care planning. So, if you're a heart failure patient in a tertiary care center and we teach you to do a certain, you'll have a video about that when you come home or when you go to a skilled nursing facility.

I should say that we have 12 hospitals, a 650-bed tertiary care center in Portland, we have four critical access hospitals, and the rest are kind of between these five affiliated skilled nursing facilities in the Portland area that are part of this program.

\section{Please describe your program? [03:17]}

What you really care about is the program model. We want to keep people at home. So, what we were looking for was ease of use-as fully automated as we can make this.

And I talked a little bit about that in terms of the interfaces that we've built. How could we integrate patient education, and kind of thinking about the areas of adult learning? We wanted to do that in multiple ways. So, could we have videos, and we could have the tablet speak to patients? That sort of thing.

We definitely wanted to look at clinical outcomes: patient satisfaction and reduced readmission. We decided, as I was going to say earlier, that we were going to look at this as a system and not worry about the reimbursement to the agency. That's going to be really crucial for people trying to set up programs, and I think you've heard that. You can think about the money, you've got to really think about, at this point, what are the outcomes you want to achieve. I'm going to talk about money, actual money that we have, dollars and cents that we can tell you about.

\section{How do you define your protocols? [04:22]}

We have a delivery model. We did several focuses of population, and what you're going to see numbers about is that we decided that heart failure was the thing that we had probably the most experience as an agency. Dr. Ester Shao was a researcher who 
actually came up with this protocol on the west coast. She got recruited probably five, six, seven years ago to MaineHealth, and she runs a heart failure clinic in our tertiary care center. So, that's where this protocol came from. We decided probably two-and-ahalf years ago or so that we would bring it into the home.

So, I should tell you that it's an acute care protocol, it's something that patients typically either use the ER (emergency room) and get admitted to the hospital for, and those are the numbers that I'm going to have for you.

There are a couple of people, and this is probably the most important slide that you need to know (Slide 4). None of this works without people that are thoroughly trained and really understand what we're doing. Again, we take technology and we throw it out we say, "Oh we're going to collect these numbers and then what that we did with them?" So, we actually have our telehealth nurses go and work at that at the heart failure clinic. They get trained as though they were nurses in the clinic and then bring that care to the patients, and that's the significant difference as to how care is delivered in this program. 


\section{Telehealth Delivery of Care Model}

Referrals received from system healthcare providers

- Population Focus: Congestive Heart Failure, Chronic Obstructive Pulmonary Disease, Cardiac Surgery, and Diabetes

MHCAH Telehealth Team reviews medical record and assesses for Telehealth admission

$>$ Home Diuretic Protocol assessment:

$>$ Frequent re-admissions for volume overload

$>$ Failed up-titrations of home diuretics

$>$ Stable renal function

$>$ Home weights correlate to volume overload

$>$ Patient demonstrates compliance

MaineHealth

CARE AT HOME

Slide 4. Telehealth delivery of care model.

How do patients use your technology? [05:41]

We did have ease of use with the technology that we have. In fact, the way the tablet is set up we've have two people in two years that have said they don't want to use it. Many people say, "What's going to happen with elderly people?" It's visually the sort of thing that people are fine with, and once we teach them how to use it, it's all touch, basically. It also talks to the patient, and it can talk to the patient in 18 different languages, so that's a real plus as well.

So, some of our results are good patient education and our outcomes (e.g., reduce admission penalties). The things we said we were going to do we actually did. 
Some of our strategies, and I think these are probably some of the more important strategies (Slide 5). We were careful about which partners we selected. We didn't go out and say we were going to do all cardiologists in Southern Maine. We actually picked a couple of cardiology practices at the hospital. We really wanted to kind of look at technology and see what we could do?

We promoted the agency; and some of the data you're going to use a lot is to just talk to folks right around our service area and say, "Here's what we can do for you." We've begun to use some of these data to talk about other types of disease problems.

We created custom materials. All of our heart failure educational material, again, is MaineHealth material, and it's loaded up either in video form or in PDF form. For those patients that want to read they can do that on their tablet, for those patients that want to listen to it, they can do that.

\section{Strategies for Enhanced Patient Engagement}

- Dignity and Respect

- Patient and family knowledge, values, beliefs and cultural backgrounds are incorporated into the planning and delivery of care.

- Information Sharing

- Patients and families receive timely, complete and accurate information in order to effectively participate in care and decision-making.

- Participation

- Patients and families are encouraged and supported in participating in care and decision-making at the level they choose.

- Collaboration

- Patients and families collaborate in policy and program development, implementation and evaluation; in professional education, as well as in the delivery of care. 
Slide 5. Strategies for patient engagement with telehealth.

\section{How do you define the work MaineHealth does? [07:14]}

We see this as interdisciplinary. So, the idea that providers are working together is really important. One of the things that happened when I came to MaineHealth two years ago; we basically did it as a nursing process, and we stopped that and said if a provider doesn't give us provider-ordered parameters, we're not going to put the patient on telehealth.

That dramatically changed accountability, because then they knew we were going to call them and we're going to ask them what to do, and if they had protocols it would be really easy to activate those protocols. So, it forced people to collaborate in a way they had not been.

\section{How does MaineHealth educate providers regarding Telehealth? [07:53]}

Educating others, so that wasn't just about providers. Senior service organizations and community organizations needed to know about this because they could also be providing services in a patient's home. Like, "What was this thing that their 90-year-old patient is suddenly using?"

We also had to create a referral system and then, as I said, we've done that in Epic. But we also have the ability to do things like faxing and voice mailing for those providers who work outside the Epic system (Slide 6). 


\section{Strategies for Collaboration with Providers}

- Partner with select providers

- Promote your agency

- Create custom materials

- Collaborate with all members of the health care team

- Educate others about what you do

- Create a referral communication system

\section{MaineHealth}

CARE AT HOME

Slide 6. Strategies for collaboration.

\section{What is the feedback from patients? [08:24]}

You heard the dignity and the respect issue, and I think that's true for this as well. We had to figure out how we would be able to share information for patients in order to have them participate, to collaborate with us. And it's very interesting for new heart failure patients on this program because they can take their tablet with them. They actually do things like take it to a restaurant. When they're going to eat there's a whole nutrition section, and they'll actually punch in the meal that they want to have and see what are the restrictions. So, it's really interactive at several different levels. It also does medication reconciliation.

\section{Outcomes [08:57]}


Here's our results for the first year of the program (Slide 7). So, March 2015 to August 2015 , for three months of the program, we set up 275 patients. There's no such thing as a pure heart failure patient. We were going to treat people for heart failure but they clearly had multiple co-morbidities then multiple secondary conditions. Improved outcomes patient endurance was up around $75 \%$ to almost $80 \%$. And that's measured by the company not by us, they're actual measures of how often people transmit their results and those sorts of things.

The patient satisfaction is a 1-4 scale, and this was what patients reported to our vendor for their satisfaction. It was really good.

We reduced every hospitalization rate for 30 days to $11.3 \%$ and for 60 days to $4.72 \%$. So, we thought we were doing really well; we had no idea how good we could really be.

\section{Our Results:}

\section{March 2015 - August 2015}

$>$ Served 275 Patients (CHF, COPD, Diabetes)

$>$ Patient Adherence: $74.5 \%-77 \%$

$>$ Improved Clinical Outcomes

$>$ Increased Patient Satisfaction Average (top 4)

- $3.91 \%$ - would recommend tablet to family/friends

- $3.81 \%$ - tablet is easy to use and positive experience

- $3.67 \%$ - tablet increases satisfaction with healthcare provider

$>$ Reduced Hospital Readmission

- 30-day $11.3 \%$

- 60-day $4.72 \%$ 
Slide 7. MaineHealth outcomes in telehealth: March through August 2015.

These are our results for October of last year to December of last year. The 30-day readmission rate is now $0.7 \%$, and our ED (emergency department) visits is zero; and the reason for that is we've become skilled, and our nurses have become skilled, at anticipating what these readings mean. The old way of looking at is, was as a twopound weight gain in a day or so much over four days or seven days, depending on the protocol. Now we're looking at weight gain over 30 days. And some things we really tightened up and figured out that trend is a lot more important for the longer term.

\section{Our Results: October 2016 - December 2016}

$>$ Served 276 Patients (CHF, COPD, Diabetes)

$>$ Patient Adherence: $74.7 \%-86.7 \%$

$>$ Improved Clinical Outcomes

$>$ Increased Patient Satisfaction Average (top 4)

- $4.08 \%$ - would recommend tablet to family/friends

- $3.78 \%$ - tablet is easy to use and positive experience

- 3.83\% - tablet increases satisfaction with healthcare provider

$>$ Reduced Hospital Readmission

- 30-day $\quad 0.7 \%$

- ED visits $0.0 \%$

Slide 8. MaineHealth outcomes in telehealth: October through December 2016. 
Our patient adherence has gone up to $86 \%$. I think part of that is how staff are able to explain how to use this a lot better and are able to kind of instruct family members to support the patients. They really kind of polish that up a lot. And we still have great patient satisfaction.

\section{How do you group your patients? [10:58]}

Here's some of this diuretic protocol (slide 9). We have three types of patients on telehealth: we have heart-failure patients on telehealth with diuretic protocol, we have heart failure patients who are on telehealth without the protocol, and we have newly diagnosed heart failure patients. So, those are three different strategies; and what I'm going to talk about are the sickest patients who would end up somehow getting care. And we had one 153 activations, and this is the first set of numbers that I showed you.

\section{MaineHealth Home Diuretic Protocol}

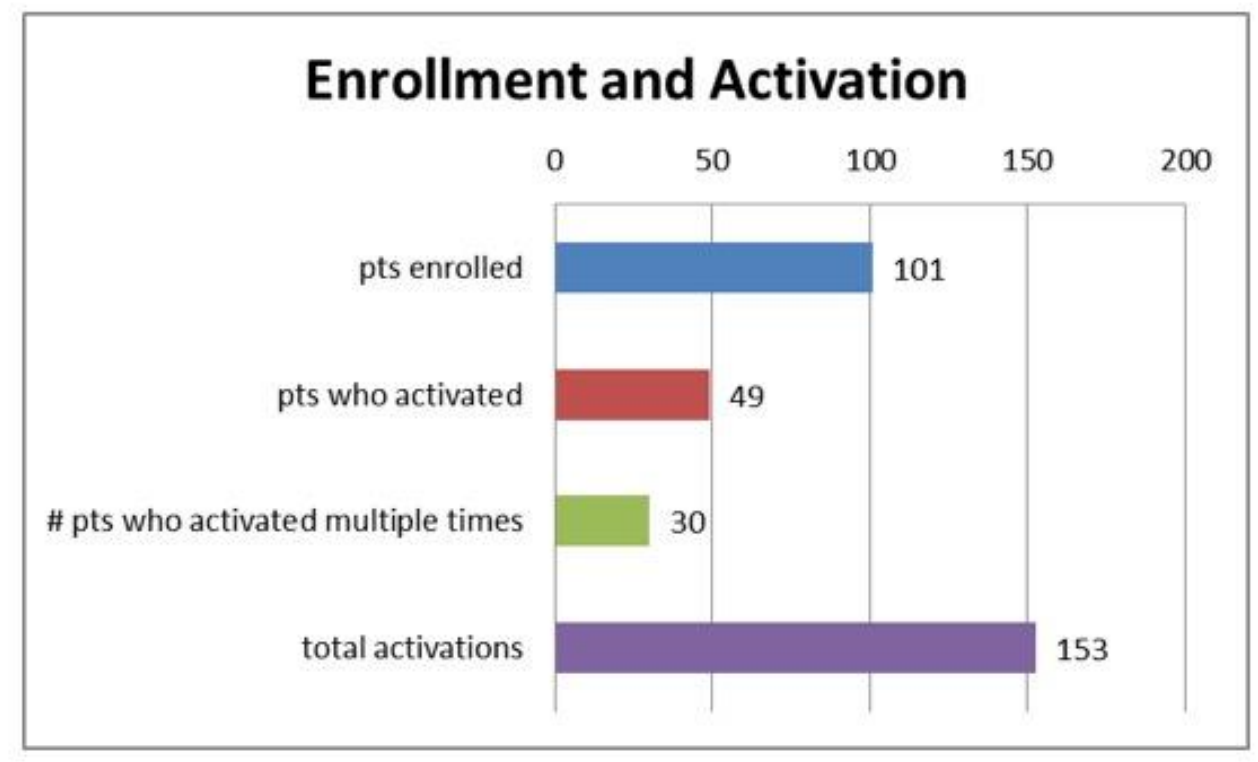

MaineHealth

Slide 9. MaineHealth diuretic protocol. 
There were 101 patients enrolled in this period, 49 were activated, and 30 were activated multiple times. So, what that means is each time there were multiple activations we prevented them from going to the ER or being admitted to the hospital.

We actually provided the care that they would have provided in the ER or in the hospital, in the home. Part of the strategy for that was we looked at what actually happened on our cardiac unit in our tertiary care center, and basically, they did exactly what this protocol says. Sometimes they would put people on a heart monitor, sometimes they wouldn't. They'd send a nurse in every day to draw blood, or a lab tech. They'd get the results, and they'd administer a diuretic, and that was basically the essential care the patient would receive for that day.

\section{What are the specifics regarding the diuretic protocol? [12:15]}

These are some of the technicalities of the protocol: doubling a diuretic, getting IV diuretics, and that sort of thing (Slide 10). What I should tell you about these numbers that you're looking at is MaineHealth actually did these numbers. We had some of our analysts, about a year ago, pull these numbers, and this had to do with a project that the ACO was sending up to the federal government, because we wanted to look at the money. 


\section{HDP Experience}

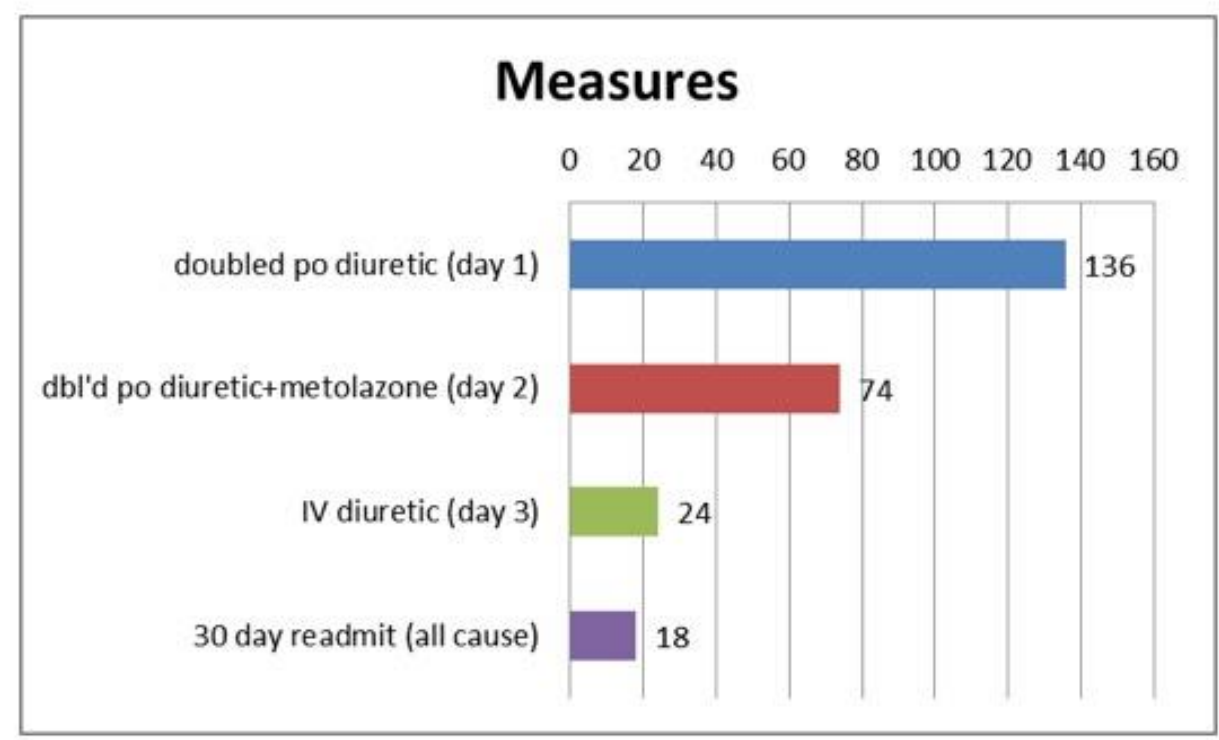

MaineHealth

CARE AT HOME

Slide 10. Details of the diuretic protocol ( $n=$ patients).

For those of you in the audience who care about this. Creatinine levels actually improved on a diuretic protocol. We were worried about what lab work was going to do and the impact of that in a community. "Were we going to make people sick in the community?" And in fact, there was no difference between what happened in the community and what happened in a facility.

The numbers that we looked at were things like average length of stay, mortality, ejection fraction. There was no difference really when we took 100 patients on the old protocol and 100 patients on the new protocol. And there really wasn't any difference in those groups. The fact that we are getting a little bit more aggressive with the care we are providing at home really, again, didn't impact any of them negatively. 


\section{How much are you saving with telehealth? [13:44]}

Here's the cost of providing care at home; about $\$ 30$ a day is what we projected for the telehealth, which is not our actual cost, but our actual cost is much cheaper than that but we had to come up with a number for the four-day period (Slide 11). So, we divided the total cost for telehealth for the month. So, it's $\$ 120$ per month. That's how we arrived at that number. How much was a nursing visit, then how much was the cost of the labs in our system and our own lab company, the IV kit (which is a one-time cost) and how much the diuretics were? The cumulative of that was $\$ 1,154.50$ for four days each year.

\section{Home Diuretic Protocol: Cost}

\begin{tabular}{|l|c|c|c|c|c|}
\multicolumn{1}{l|}{ HDP } & Baseline & Day 1 & Day 2 & Day 3 & Day 4 \\
\hline telehealth & $\$ 30.00$ & $\$ 30.00$ & $\$ 30.00$ & $\$ 30.00$ & $\$ 30.00$ \\
\hline nurse visit & $\$ 162.95$ & $\$ 162.95$ & $\$ 162.95$ & $\$ 162.95$ & $\$ 162.95$ \\
\hline labs & $\$ 31.95$ & $\$ 31.95$ & $\$ 31.95$ & $\$ 31.95$ & $\$ 31.95$ \\
\hline IV kit & $\$ 25.00$ & $\$ 0.00$ & $\$ 0.00$ & $\$ 0.00$ & $\$ 0.00$ \\
\hline IV diuretic & $\$ 0.00$ & $\$ 0.00$ & $\$ 0.00$ & $\$ 5.00$ & $\$ 0.00$ \\
\hline total & $\$ 249.90$ & $\$ 224.90$ & $\$ 224.90$ & $\$ 229.90$ & $\$ 224.90$ \\
\hline cumulative & & $\$ 474.80$ & $\$ 699.70$ & $\$ 929.60$ & $\$ 1,154.50$ \\
\hline
\end{tabular}

MaineHealth

CARE AT HOME

Slide 11. Diuretic protocol cost. 
Then we looked at the actual care of just delivering the telehealth with a nursing visit, so that's $\$ 475$ (Slide 12).

\section{Home Diuretic Protocol: Cost}

Comparison Group

\begin{tabular}{|l|c|c|c|c|c|}
\multicolumn{1}{l|}{} & Baseline & Day 1 & Day 2 & Day 3 & Day 4 \\
\hline telehealth & $\$ 30.00$ & $\$ 30.00$ & $\$ 30.00$ & $\$ 30.00$ & $\$ 30.00$ \\
\hline nurse visit & $\$ 162.95$ & & & & $\$ 162.95$ \\
\hline total & $\$ 192.95$ & $\$ 30.00$ & $\$ 30.00$ & $\$ 30.00$ & $\$ 192.95$ \\
cumulative & & $\$ 222.95$ & $\$ 252.95$ & $\$ 282.95$ & $\mathbf{\$ 2 7 5 . 9 0}$
\end{tabular}

Slide 12. Home diuretic protocol: comparison group cost.

To go to the ED, which some patients were doing, for four days and getting the same set of lab work and the same treatment was $\$ 7,137$. And then for that group of patients that got admitted —and, at the point that we started this, there were a fair amount that were getting admitted for four days to receive this-was $\$ 43,172$ for the exact same care (Slide 13). 


\section{Home Diuretic Protocol: Cost}

\begin{tabular}{|c|c|c|c|}
\hline \multicolumn{4}{|c|}{$\begin{array}{l}\text { Maine Medical Center ED and Hospitalization for } \\
\text { Heart Failure: Jan-Mar } 2015\end{array}$} \\
\hline & $\begin{array}{c}\text { all } \\
(1025)\end{array}$ & $\begin{array}{l}\text { discharged } \\
(728)\end{array}$ & $\begin{array}{l}\text { admitted } \\
(279)\end{array}$ \\
\hline Avg ED charges & $\$ 2,837.00$ & $\$ 1,644.00$ & $\$ 3,272.00$ \\
\hline Avg hospital charges & $\$ 30,632.00$ & $\$ 5,493.00$ & $\$ 39,900.00$ \\
\hline \multicolumn{2}{|l|}{ total } & \multicolumn{2}{|l|}{$\$ 7,137.00$} \\
\hline
\end{tabular}

MaineHealth

CARE AT HOME

Slide 12. Home diuretic protocol: hospitalization cost.

The other thing the analysts did is they went back into the record and looked at the care the patient actually received to be sure that there weren't any discrepancies. And there really wasn't anything significant that was happening in the in-patient admission.

A couple things that I want to tell you about, and then we can have questions. One is that when we looked at rehospitalization and we looked at the cost. If you think about that $\$ 44,000$ versus the $\$ 1,00$ and 151 activations that we did, we saved 151 times $\$ 42,000$ in that six-month period. That's really significant, and who that was significant to was our ACO because once the patient gets admitted, either to the ED or any facility, they have no control over the costs for that. 
We decided as a system that it was worth the home health agency taking the hit, if there was a hit, and it really isn't because we can pretty well get reimbursed for most of this care, and the patients typically pay for the meds. So, there's not really any hit for it right now. But as an organization we decided that it was better if the home health agency had to take a hit for the ACO to save that $\$ 42,000$ for each of those activations.

\section{How do you adapt your system to individual patients? [16:13]}

Now understand it doesn't work for everybody, and we have a great integrated cooperative way that our entities work together. That doesn't work for every system, I get that. But I think there is a really good argument to be made that you really should work better with your home health agencies. The other message I want to bring, which we had talked about earlier, so we are doing this in SNFs (skilled nursing care facilities) and we are getting the exact same results.

Actually, we're probably getting better results in the SNF than we are in home health because the patients are already in a supervised situation; and so, the chances of them getting activated at all are actually a little bit lower. Medicare is paying typically for that first-time period of them being in this SNF.

So, it's really interesting. We have a MaineHealth physician who is the Medical Director of one facility in particular who has really championed this since, they have become our direct partner; they get the patient from the hospital and then we take them home, we bring the equipment into the SNF that they're going to be using at home and that's how the patient gets introduced to it.

Question: In the skilled nursing facility are you putting the technology in there or just the protocol from day one or just before? [17:27]

Mr. Abel: The SNF has their own, so they're not using telehealth specifically but they're using a tablet to stream their own system; but then when we know the patient is going to be discharged we'll bring our own technology in. 
So, the patient becomes familiar with that before they get home. One of the things we've really learned is weight is probably the single most important indicator for us to pay attention to. So, we want the weight to be done in the facility on our equipment for a couple of days so we show we have the right number.

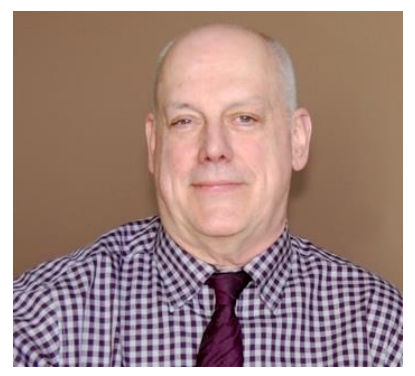

Robert Abel, MSN, RN, CHPN, CMC, CCM, is the Chief Nursing Officer for MaineHealth Care at Home. He has responsibility for the agency's telehealth program and involved in several system-wide initiatives to implement telehealth programs in areas such as heart failure, palliative care, and pediatrics. Mr. Abel previously worked with interdisciplinary clinical programs that used telehealth for decentralized, team-based, disease-driven care models.

Tags: Robert Abel, COPD, economics of telehealth, governance, heart failure, home care, MaineHealth, Medicare, nursing care, sustainability, telehealth 